\title{
An extramedullary plasmacytoma in the testicle: A case report and review of the literature
}

\author{
Kathy Lue, BS; ${ }^{*}$ Justin B. Emtage, MD; Maria Ana Pariñas, BScN, RN;; Jasreman Dhillon, MD;* \\ Julio Pow-Sang, $M D^{f}$
}

*University of South Florida, College of Medicine, Tampa, FL; 'Department of Urology, University of South Florida, Tampa, FL; §Department of Nursing, Florida Hospital Tampa, Tampa, FL; *Department of Anatomic Pathology, Genitourinary, Moffitt Cancer Center, Tampa, FL; £Department of Genitourinary Oncology, Moffitt Cancer Center, Tampa, FL

Cite as: Can Urol Assoc J 2015;9(3-4):E240-2. http://dx.doi.org/10.5489/cuai.2531

Published online April 13, 2015.

\begin{abstract}
We present a rare case of testicular extramedullary plasmacytoma (EMP) in a 43-year-old man with multiple myeloma and diffuse systemic involvement refractory to chemotherapy. Multiple myeloma is typically found within the bone marrow and rarely involves other organs and sites. EMPs are most frequently associated with the head and neck region, but in rare cases testicular involvement have been seen. These mimic other causes of testicular swelling and therefore require a full diagnostic workup and management similar to that of any scrotal pathology. Radical inguinal orchiectomy is the treatment of choice with the addition of adjunct or salvage radiation therapy if necessary. The prognosis with these lesions is poor and in cases of primary testicular plasmacytoma, progression to multiple myeloma is likely.
\end{abstract}

\section{Introduction}

Multiple myeloma is a rare neoplastic disorder characterized by malignant proliferation of plasma cells within the body's bone marrow. Multiple myeloma comprises about $1 \%$ of all cancers and classically presents with hypercalcemia, renal function impairment, anemia, and osteolytic bone lesions. Although typically found within the bone marrow, extraosseous myelomatous collections referred to as extramedullary plasmacytomas (EMPs) are rarely found in the head and neck region, gastrointestinal tract, and central nervous system. ${ }^{1}$ They can either be primary lesions or indicators of progressive disease. ${ }^{2,3}$ Testicles are an extremely rare site for EMP involvement. Following the first reported case in 1939, less than 70 of these events have been described in the literature. ${ }^{4,5}$ Among testicular masses, plasmacytoma is even more rare, with an overall incidence of $0.03 \%$ to $0.1 \% .6,7$ Due to the rarity of presentation, published literature is limited, yet it has an extremely poor prognosis. ${ }^{8,9}$

\section{Case report}

A 43-year-old man, with a 3-year history of diffusely systemic multiple myeloma refractory to radiation and chemotherapy, presented to our facility with 4-week history of continuous, painless left testicular swelling. Two weeks prior, he was seen at a local emergency department with a presumed diagnosis of left orchitis. However, following a prescribed 2-week course of oral ciprofloxacin, the patient noted no resolution of his symptoms. On physical exam, the patient had an enlarged $(8.5 \times 4.5 \times 5 \mathrm{~cm})$ non-tender left testicle and a normal-appearing right testicle. Laboratory values were not significant for an abnormal urinalysis or white blood cell count. Testicular tumour markers ( -fetoprotein, $\beta$-hCG and lactate dehydrogenase) were within normal limits. Testicular ultrasound showed a diffusely enlarged heterogeneously echogenic left testicle and epididymis with significant hyperemia (Fig. 1).

Given the concerning scrotal ultrasound and lack of suggestive evidence of infection, the patient agreed to undergo a left radical inguinal orchiectomy for suspected malignancy. Gross examination revealed a roughly 8-cm soft, tan-coloured fleshy homogenous mass. Pathology showed plasma cell myeloma with involvement of the testicular parenchyma, tunica albuginea and spermatic cord. Tumour cells were present in diffuse sheets almost completely replacing the normal testicular parenchyma. Histologic examination revealed discohesive, round to oval cells with a moderate amount of cytoplasm, and single nuclei focally binucleated with a single prominent nucleolus. Immunohistochemical stains were positive for lambda light chain and CD138, and negative for kappa light chain, placental alkaline phosphatase, and CD117. CD20 staining exhibited a non-specific, patchy background (Fig. 2). 


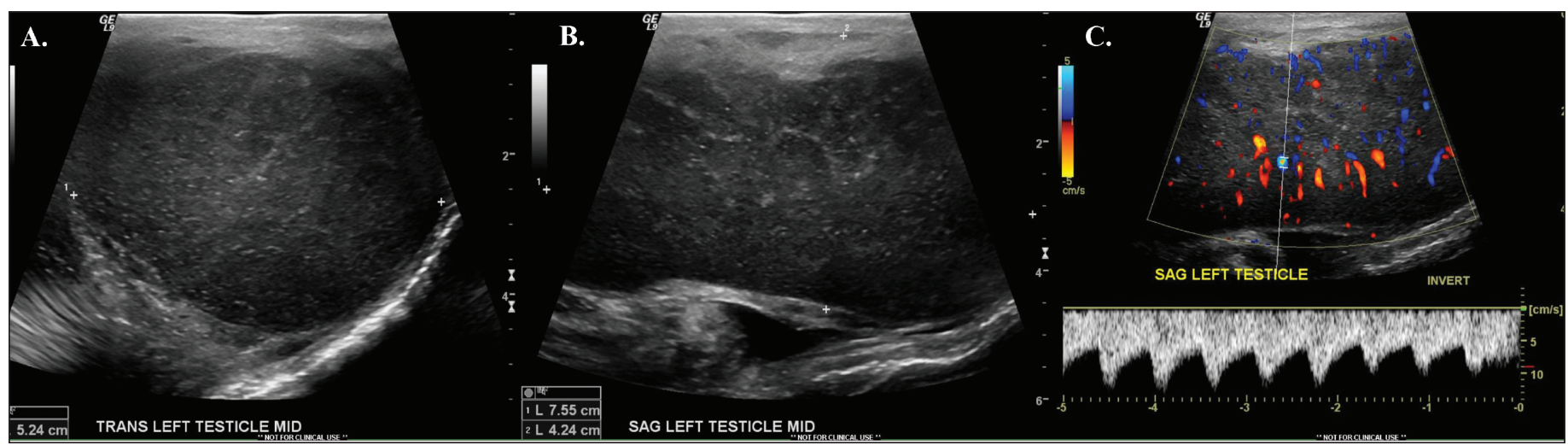

Fig. 1. Scrotal ultrasound showing a diffusely enlarged heterogeneously echogenic left testicle with increased flow. (A) Transverse view of the mid left testicle. (B) Sagittal view of the mid left testicle. (C) Sagittal view of the mid left testicle with Doppler.

\section{Discussion}

Testicular plasmacytomas have been identified in multiple settings, most involving patients with concurrent multiple myeloma and diffuse disease involvement of other organs, as in our present case. ${ }^{10-12}$ Testicular EMPs have also been reported as a site of recurrence during multiple myeloma remission. ${ }^{13}$ This is thought to be secondary to the bloodtestes barrier creating a haven for tumour formation in the testicle. In rare cases, plasmacytoma of the testes can occur in the absence of documented hematologic malignancy. ${ }^{9,11,12}$ Unfortunately, most of these patients will develop multiple myeloma, with only a few long-term progression-free survivors post-orchiectomy. ${ }^{14}$

As with primary testicular masses, radical inguinal orchiectomy is the preferred surgical treatment. These tumours are markedly radiosensitive and therefore may response well to adjuvant and/or salvage radiation therapy. ${ }^{15}$ Despite advancements in treatment options, the prognosis for affect- ed patients continues to be poor. Anghel and colleagues performed a literature review of testicular plasmacytoma cases and previous or concurrent multiple myeloma or EMP to assess survival. In their assessment, $59 \%$ of patients died within 26 months of diagnosis, with $60 \%$ of deaths occurring within the first 12 months post-orchiectomy. ${ }^{10}$

Our case included a variety of interesting factors. Historically, the average age of testicular plasmacytoma onset is about 55 to 60 years, while our patient was relatively young (age 43). The rate of testicular enlargement was noticeably rapid over a 3 -week period and inconsistent with the insidious growth pattern reported previously. ${ }^{11,12}$ This lends to an especially aggressive disease given its progression and how refractory it was to multiple treatment modalities. The predominant immunoglobulin subtype seen in patients who have multiple myeloma and testicular EMPs is $\lg A$, which is consistent with our case findings. ${ }^{10}$ Previous reports have questioned whether the IgA subtype lends to a more aggressive course. ${ }^{9}$ With further follow-up,

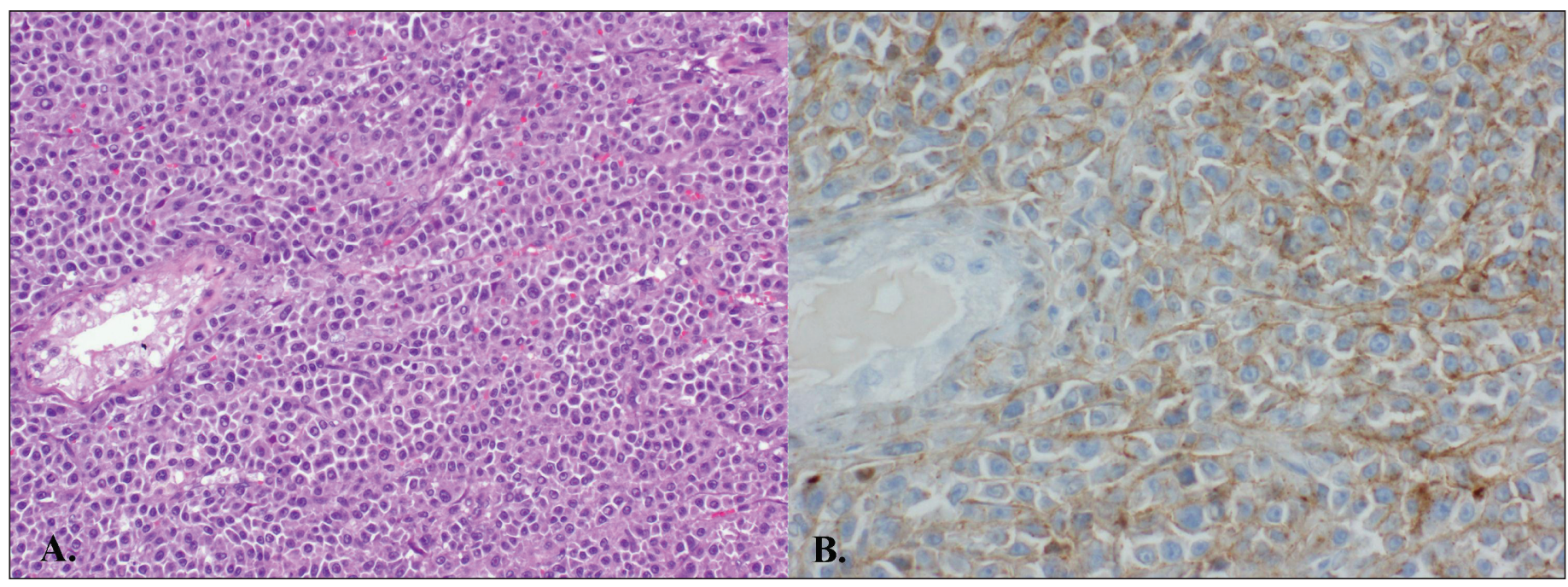

Fig. 2. Histologic slides of the left testicle specimen. (A) Hematoxylin and eosin stain showing diffuse infiltration of plasma cells around a seminiferous tubule. (B) Diffuse positivity for Immunohistochemical stain CD138 in the plasma cells. 
Lue et al.

our case could contribute to this investigation. Analyzing a larger prospective cohort would be ideal to appropriately determine an association with immunoglobulins and other prognostic factors. Although its rarity makes this unlikely, such a study would help guide management, improve collaborative decision making, and enhance the potential for an improved quality of life.

\section{Conclusion}

We present a case of testicular EMP presenting with multiple myeloma and diffuse systemic involvement. EMPs are most frequently associated with the head and neck region, but in rare cases testicular involvement has been seen. These mimic other causes of testicular swelling and therefore require a full diagnostic workup and management similar to that of any scrotal pathology. Radical inguinal orchiectomy is the treatment of choice, but radiation therapy can be used as an adjunct or salvage. The prognosis with these lesions is poor and in cases of primary testicular plasmacytoma, progression to multiple myeloma is likely.

Competing interests: The authors declare no competing financial or personal interests.

This paper has been peer-reviewed.

\section{References}

1. Kapadia SB. Multiple myeloma: A clinicopathologic study of 62 consecutively autopsied cases. Medicine 1980;59:380-92. http://dx.doi.org/10.1097/00005792-198009000-00005

2. Hayes DW, Bennett WA, Heck FJ. Extramedullary lesions in multiple myeloma: Review of literature and pathologic studies. AMA Arch Pathol 1952;53:262-72.

3. Pasmantier MW, Azar HA. Extraskeletal spread in multiple plasma cell myeloma. A review of 57 autopsied cases. Cancer 1969;23:167-74. http://dx.doi.org/10.1002/1097-0142(196901)23:1<167::AIDCNCR2820230122>3.0.C0;2-0

4. Wang YM, Li FY, Luo JD, et al. Testicular plasmacytoma: A case report and review of the literature. Chin Med J 2008;121:956-8.

5. Ulrich H. Multiple myeloma. Arch Intern Med 1939;64:994.

6. Levin HS, Mostofi FK. Symptomatic plasmacytoma of the testis. Cancer 1970;25:1193-203. http:// dx.doi.org/10.1002/1097-0142(197005)25:5<1193::AID-CNCR2820250527>3.0.C0;2-6

7. Chica $G$, Johnson DE, Ayala AG. Plasmacytoma of testis presenting as primary testicular tumor. Urology 1978;11:90-2. http://dx.doi.org/10.1016/0090-4295(78)90213-3

8. Avitable AM, Gansler TS, Tomaszewski JE, et al. Testicular plasmacytoma. Urology 1989;34:51-4. http:// dx.doi.org/10.1016/0090-4295(89)90159-3

9. Hathaway AR. Incidental discovery of a testicular plasmacytoma at initial presentation of multiple myeloma. Case Rep Hematol 2013;2013:752921. http://dx.doi.org/10.1155/2013/752921

10. Anghel $G$, Petti N, Remotti D, et al. Testicular plasmacytoma: Report of a case and review of the literature. Am J Hematol 2002;71:98-104. http://dx.doi.org/10.1002/aih.10174

11. Ferry JA, Young RH, Scully RE. Testicular and epididymal plasmacytoma: A report of 7 cases, including three that were the initial manifestation of plasma cell myeloma. Am I Surg Pathol 1997;21:590-8. http://dx.doi.org/10.1097/00000478-199705000-00013

12. Melicow MM, Cahill GF. Plasmacytoma (multiple myeloma) of testis: A report of four cases and review of the literature. J Urol 1954;71:103-13.

13. Rosenberg S, Shapur N, Gofrit 0, et al. Plasmacytoma of the testis in a patient with previous multiple myeloma: Is the testis a sanctuary site? J Clin Oncol 2010;28:e456-8. http://dx.doi.org/10.1200/ JC0.2009.27.6519

14. Filho MG. Primary testicular plasmocytoma: A five year follow-up. Urol Annal 2013;5:39-41. http:// dx.doi.org/10.4103/0974-7796.106965

15. Alexiou C, Kau RJ, Dietzfelbinger H, et al. Extramedullary plasmacytoma: Tumor occurrence and therapeutic concepts. Cancer 1999;85:2305-14. http://dx.doi.org/10.1002/(SICI) 10970142(19990601)85:11<2305::AID-CNCR2>3.0.C0;2-3

Correspondence: Dr. Justin B. Emtage, Department of Urology, University of South Florida, South Tampa Center for Advanced Healthcare, 2 Tampa General Circle, 7th Floor, Tampa, FL 33606; jemtage@health.usf.edu 